\title{
Tolerance to Residual Refractive Errors After Trifocal and Trifocal Toric Intraocular Lens Implantation
}

\author{
Laureano A. Rementeria-Capelo, M.D., Inés Contreras, M.D., Ph.D., Jorge L. García-Perez, M.D., Ph.D., \\ Virginia Carrillo, O.D., Juan Gros-Otero, M.D., Ph.D., and Javier Ruiz-Alcocer, Ph.D.
}

\begin{abstract}
Objective: The objective of this study was to assess the impact of 0.50 diopter (D) positive or negative defocus on visual function in patients implanted with trifocal and trifocal toric intraocular lenses (IOLs).

Methods: The study included patients implanted with the AcrySof IQ PanOptix IOL or the PanOptix Toric. Visual acuity (VA) at high (100\%), medium $(50 \%)$ and low (10\%) contrast, contrast sensitivity function (CSF), and halo perception were assessed three months after surgery. Explorations were performed with corrected distance visual acuity (CDVA), with a positive defocus of $+0.50 \mathrm{D}$ (myopization) and with a negative defocus of $-0.50 \mathrm{D}$ (hyperopization).

Results: The study included 60 eyes of 60 patients (30 eyes with PanOptix and 30 eyes with PanOptix Toric). For both groups, VA was better for all contrast settings at the CDVA situation $(P<0.05$ in all cases). For low spatial frequencies, no differences in CSF were found among the three refractive situations in either group. For higher frequencies, the results showed an overall trend for better CSF results for the CDVA situation. The halo effect was lower for the CDVA situation if compared to myopization and hyperopization in both the PanOptix and the PanOptix Toric groups $(P<0.05$ for all cases).

Conclusions: There is an impact on visual quality and halo perception in patients implanted with trifocal or trifocal toric IOLs even for low residual refractive errors.
\end{abstract}

Key Words: Defocus-Trifocal-Trifocal toric-Intraocular lensesPhotic phenomena.

(Eye \& Contact Lens 2020;00: 1-6)

$\mathrm{T}$ rifocal and trifocal toric intraocular lenses (IOLs) have become popular among cataract surgeons due to the satisfactory clinical outcomes and the high rates of spectacle independence achieved by their patients. ${ }^{1-3}$ However, to reach optimal visual results, residual refraction should be close to emmetropia; otherwise, residual refractive errors might reduce visual quality and increase the rate of dysphotopsias.

From the Clínica Rementería (L.A.R.-C., I.C., J.L.G.-P., V.C., J.G.-O.), Madrid, Spain; Hospital Universitario Ramón y Cajal (I.C.), Madrid, Spain; Instituto Ramón y Cajal de Investigaciones Sanitarias (IRYCIS) (I.C.), Madrid, Spain; and Departamento de Optometría y Visión, Universidad Complutense de Madrid (J.R.-A.), Madrid, Spain.

The authors have no funding or conflicts of interest to disclose.

Address correspondence to Javier Ruiz-Alcocer, Ph.D., Optometry and Vision, Department Faculty of Optics and Optometry, Universidad Complutense de Madrid, C/Arcos de Jalón, 28037 Madrid, Spain; e-mail: jruizalcocer@ucm.es

Accepted May 17, 2020.

DOI: 10.1097/ICL.0000000000000726
Different studies have reported excellent refractive outcomes after the implantation of trifocal IOLs, with approximately $95 \%$ of patients with spherical equivalent (SE) values within \pm 0.50 diopters (D) of emmetropia, ${ }^{2,4-6}$ although other authors reported lower values. ${ }^{4,7}$ In patients with residual refractive errors that have an important effect on visual function, corneal refractive surgery may be performed with good clinical outcomes. ${ }^{8,9}$ Nonetheless, it is difficult to accurately correct residual refractions of less than $0.50 \mathrm{D}$.

Therefore, it would be of interest to evaluate the visual performance of patients who have received a trifocal IOL who have small refractive errors. One of the ways to do this is to induce either myopia or hyperopia over an ideal emmetropic situation. Thus, the aim of this study is to assess visual quality and halo perception when a certain amount $(0.50 \mathrm{D})$ of positive or negative defocus is induced in patients implanted with trifocal and trifocal toric IOLs.

\section{PATIENTS AND METHODS}

This prospective study was performed at Clínica Rementería, Madrid, Spain, and comprised patients who were looking for spectacle independence after cataract surgery and scheduled for bilateral implantation of the same diffractive trifocal IOL. Only one eye of each patient was evaluated in this study. The study followed the tenets of the Declaration of Helsinki and was reviewed and approved by the pertinent ethics committee. Informed consent was obtained from all patients after the nature of the study had been explained.

Exclusion criteria included amblyopia, preoperative angle kappa greater than $0.4 \mathrm{~mm}$, previous ocular surgery, and presence of ocular pathologies and abnormal iris. Patients with intraoperative or postoperative complications and with residual SE over $0.50 \mathrm{D}$ were also excluded.

The study evaluated two groups of patients. One group included eyes that were implanted with the nontoric trifocal AcrySof IQ PanOptix IOL (Alcon Laboratories, Fort Worth, TX). The AcrySof IQ PanOptix has been described in detail in previous studies. ${ }^{1,6}$ In summary, this IOL has a diffractive design with a negative spherical aberration $(-0.1 \mu \mathrm{m})$ in the anterior surface. It creates three effective foci at far, intermediate $(60 \mathrm{~cm} /+2.17 \mathrm{D}$ add $)$, and near distance $(40 \mathrm{~cm} /+3.25 \mathrm{D}) .{ }^{1,6}$ The hydrophobic acrylic material of the IOL incorporates ultraviolet and blue light filtration.

The other group of eyes received a toric trifocal AcrySof IQ PanOptix Toric (Alcon Laboratories). The AcrySof IQ PanOptix Toric shares the same design and material with the spherical 
version. However, to correct corneal astigmatism, this lens has a biconical posterior surface. It can correct up to $3.75 \mathrm{D}$ of astigmatism. ${ }^{1}$

All patients underwent an extensive ophthalmologic examination before surgery, including refraction and visual acuity (VA) measurement, slitlamp biomicroscopy, corneal topography, optical coherence tomography of the macula and optic nerve, and fundus examination under pharmacological mydriasis. Angle kappa was measured with the Pentacam HR (Oculus, Wetzlar, Germany). At the same time, patients were examined with the IOLMaster 700 for IOL calculation. Patients with more than $0.8 \mathrm{D}$ astigmatism in corneal topography were also examined with the VERION Image Guided System (Alcon Laboratories). ${ }^{1}$ Toric IOLs were implanted in eyes with more than $0.8 \mathrm{D}$ astigmatism.

All cataract surgeries were performed by two experienced surgeons (LA.R.-C. and J.L.G.-P.) under topical and intracameral anesthesia, with a $2.2-\mathrm{mm}$ clear-cornea incision at $135^{\circ}$ and "stop and chop" phacoemulsification. Toric IOL implantation was guided by the VERION System.

All patients were evaluated one day, one week, one month, and three months after surgery, but only data from the third-month visit were taken into consideration for this study. In the three-month visit, patients included in the study underwent VA for different contrasts, contrast sensitivity, and halo size evaluation. Initially, uncorrected distance VA with an ETDRS chart was measured, followed by subjective refraction (best distance correction). All other measurements were then performed first with the best distance correction and then with $+0.50 \mathrm{D}$ (myopization) and $-0.50 \mathrm{D}$ defocus (hyperopization) over this best distancecorrected situation (as the reference situation).

The different contrast VA evaluation procedures were performed with the FrACT3.9.9a version of the Freiburg Acuity Test software package. ${ }^{10,11}$ Acuity software was run on a separate laptop screen that was calibrated to be presented at 4 meters. A black Landolt $\mathrm{C}$ was presented to the subjects and, among eight different possibilities, they indicated the orientation of the optotype on a numeric keypad. This test minimizes the observer's bias because the presented optotype depends on the patient's previous responses. That is, when the observer responds to a certain optotype, the software automatically modifies the size of the next optotype according to parameter estimation by a sequential test method. Monocular VA was evaluated at low (10\%), medium (50\%), and high $(100 \%)$ contrast.

Monocular contrast sensitivity function (CSF) was measured for spatial frequencies of $3,6,12$, and 18 cycles per degree (cpd) using the functional acuity contrast test (Test SV-1000) of the CC-100 HW 5.0 Series system. Contrast sensitivity function values were analyzed as Absolute $\log 10(\log 10 \mathrm{CS})$.

The halo size perceived by patients in response to glare conditions was analyzed through the Halo v1.0 (Laboratory of Vision Sciences and Applications, University of Granada, Spain). The Halo v1.0 is an open-access software package that has been used in previous investigations that included multifocal IOLs analysis. ${ }^{12-16}$ This test was also performed with a laptop screen at $4 \mathrm{~m}$ and the measurements were performed monocularly under scotopic conditions. The software calculates a "discrimination index" (a numerical value) depending on the size of the halo reported. ${ }^{13}$ This index ranges from zero to one in decimal scale where the higher index represents a lesser halo impact.
Data analysis was performed using SPSS for Windows V.20.0 (SPSS, Inc., Chicago, IL). The normal distribution of variables was assessed using the Kolmogorov-Smirnov test. A repeatedmeasures analysis of variance was used to gauge any statistically significant difference within the different situations. Post hoc multiple comparison testing was performed using the Holm-Sidak method. Differences were considered to be statistically significant when the $P$ value was less than 0.05 (i.e., at the $5 \%$ level).

\section{RESULTS}

A total of 60 eyes of 60 patients were included in this study: 30 eyes implanted with a PanOptix IOL of 30 patients with a mean age of $67.15 \pm 10.60$ years and 30 eyes implanted with PanOptix Toric IOL of 30 patients with a mean age of $66.33 \pm 7.96$ years $(P=0.46)$. Demographic preoperative data of each group are shown in Table 1. Furthermore, all surgeries were successfully performed and there were no intraoperative or postoperative complications.

\section{Visual Acuity at Different Contrast Levels}

Distance VA values at the different measured contrast levels are recorded in Figure 1. For both the spherical group and the toric group, VA for all contrast settings was better when measured with best-corrected distance visual acuity (CDVA) compared with both defocus situations, with no significant differences between +0.50 and -0.50 defocus.

Figure 2 shows the proportion of eyes in which $100 \%$ contrast VA dropped two or more lines with 0.50D defocus. For the PanOptix group (Fig. 2A), when a positive defocus was induced, 37\% of the eyes lost $\geq 2$ lines of vision. When a negative defocus was induced, the proportion of eyes that lost $\geq 2$ lines of vision was $33 \%$. In the toric group (Fig. 2B), $42 \%$ and $50 \%$ of the eyes lost $\geq 2$ lines of vision when $+0.50 \mathrm{D}$ and $-0.50 \mathrm{D}$ of defocus were induced, respectively.

\section{Contrast Sensitivity Function}

Figure 3 shows CSF results for both groups and for all situations. Regarding the PanOptix group (Fig. 3A), for the lower spatial frequency ( 3 cycles per degree), no statistically significant differences were found between best distance correction, $+0.50 \mathrm{D}$ and $-0.50 \mathrm{D}$ defocus $(P=0.16)$. At six cycles per degree, better contrast sensitivity was found with the best distance correction, although a post hoc comparison showed that only the difference with +0.50 defocus was statistically significant $(P=0.02)$. At 12 cycles per degree, better results were again recorded with the best distance correction compared with the myopic $(P=0.01)$ and the hyperopic situations $(P=0.01)$ with no significant differences between both defocus situations $(P=0.19)$. Finally, for the highest spatial frequency (18 cycles per degree), the overall results were better with best distance correction, although the difference was statistically significant only compared to $+0.50 \mathrm{D}$ defocus $(P=0.01)$. For the toric group (Fig. 3B), the CSF results followed a very similar pattern. For three cycles per degree, the three focal positions showed similar results $(P=0.09)$. For six cycles per degree, better contrast was found with best distance correction, although the difference was only statistically significant when compared with $+0.50 \mathrm{D}$ defocus $(P<0.001)$. For the mid-high frequency of 12 cycles per degree, contrast sensitivity was better with the best distance correction compared to both defocus 
TABLE 1. Preoperative Characteristics of the Eyes Included in the Study

\begin{tabular}{lccccc}
\hline & No. of Eyes & Photopic Pupil $(\mathrm{mm})$ & Sphere (D) & Cylinder (D) & Spherical Equivalent (D) \\
\hline PanOptix & 30 & $3.39 \pm 0.96$ & $-0.98 \pm 3.10$ & $-0.54 \pm 0.45$ & $-1.25 \pm 3.13$ \\
PanOptix Toric & 30 & $3.43 \pm 1.17$ & $0.03 \pm 3.56$ & $-1.12 \pm 0.51$ & $0.18 \pm 0.27$ \\
$P$ & & 0.44 & 0.11 & $<0.001$ & $0.16 \pm 0.19$ \\
\hline
\end{tabular}

Values provided are Mean \pm SD.

CDVA, corrected distance visual acuity; LogMAR, logarithm of the minimum angle of resolution.

situations $(P=0.03$ and $P=0.01$ for $+0.50 \mathrm{D}$ and $-0.50 \mathrm{D}$, respectively). For this spatial frequency, no differences were found between $+0.50 \mathrm{D}$ and $-0.50 \mathrm{D}$ defocus $(P=0.24)$. For the highest spatial frequency (18 cycles per degree), contrast sensitivity was statistically higher with best distance correction only when compared with $-0.50 \mathrm{D}$ defocus $(P=0.02)$.

\section{Halo Effect-Index of Discrimination}

Figure 4 shows the halo results (index of discrimination) for both study groups. In both groups, the highest discrimination index (lowest halo effect) was found with the best distance correction compared to both defocus situations $(P=0.01$ for $+0.50 \mathrm{D}$ and $P=0.03$ for $-0.50 \mathrm{D}$ in the PanOptix group and $P<0.001$ for $+0.50 \mathrm{D}$ and $P<0.001$ for $-0.50 \mathrm{D}$ in the PanOptix Toric group), with no statistically significant differences between both defocus situations $(P=0.13$ for the PanOptix group and $P=0.37$ for the PanOptix Toric group, respectively).

To properly assess the impact of residual refractive errors on halo perception, Figure 5 shows the proportion of patients in both groups that, after inducing $+0.50 \mathrm{D}$ or $-0.50 \mathrm{D}$ defocus, showed a loss $\geq 10 \%$ of discrimination index (potential complains due to halo perception or due to a significant VA loss) as well as those that showed a loss $\geq 10 \%$ of discrimination index while their VA remained stable (patients who might complain only due to photic phenomena). For the PanOptix group (Fig. 5A), 30\% eyes lost $\geq 10 \%$ of the discrimination index and $17 \%$ lost $\geq 10 \%$ of the discrimination index without a VA drop with +0.50 defocus (myopization). When $-0.50 \mathrm{D}$ defocus was induced (hyperopization), $16 \%$ of eyes lost $\geq 10 \%$ of the discrimination index and $13 \%$ lost $\geq 10 \%$ of the discrimination index without a VA drop. For the PanOptix Toric group (Fig. 5B), 38\% of eyes lost $\geq 10 \%$ of the discrimination index and $15 \%$ lost $\geq 10 \%$ of the discrimination index without a VA drop with +0.50 defocus. With -0.50 defocus, $26 \%$ of eyes lost $\geq 10 \%$ of the discrimination index and $11 \%$ lost $\geq 10 \%$ of the discrimination index with a stable VA.

\section{DISCUSSION}

Inaccurate IOL calculation before cataract surgery might lead to residual refractive errors and for Premium IOLs, this could lead to decreased visual quality. ${ }^{17}$ The question is whether small refractive errors, which have been traditionally regarded as successful refractive outcomes, might have a significant impact on visual function and therefore on patients' satisfaction. Common complaints in patients after multifocal IOL implantation, with good visual acuities measured in an office, are the need for a potent light source to
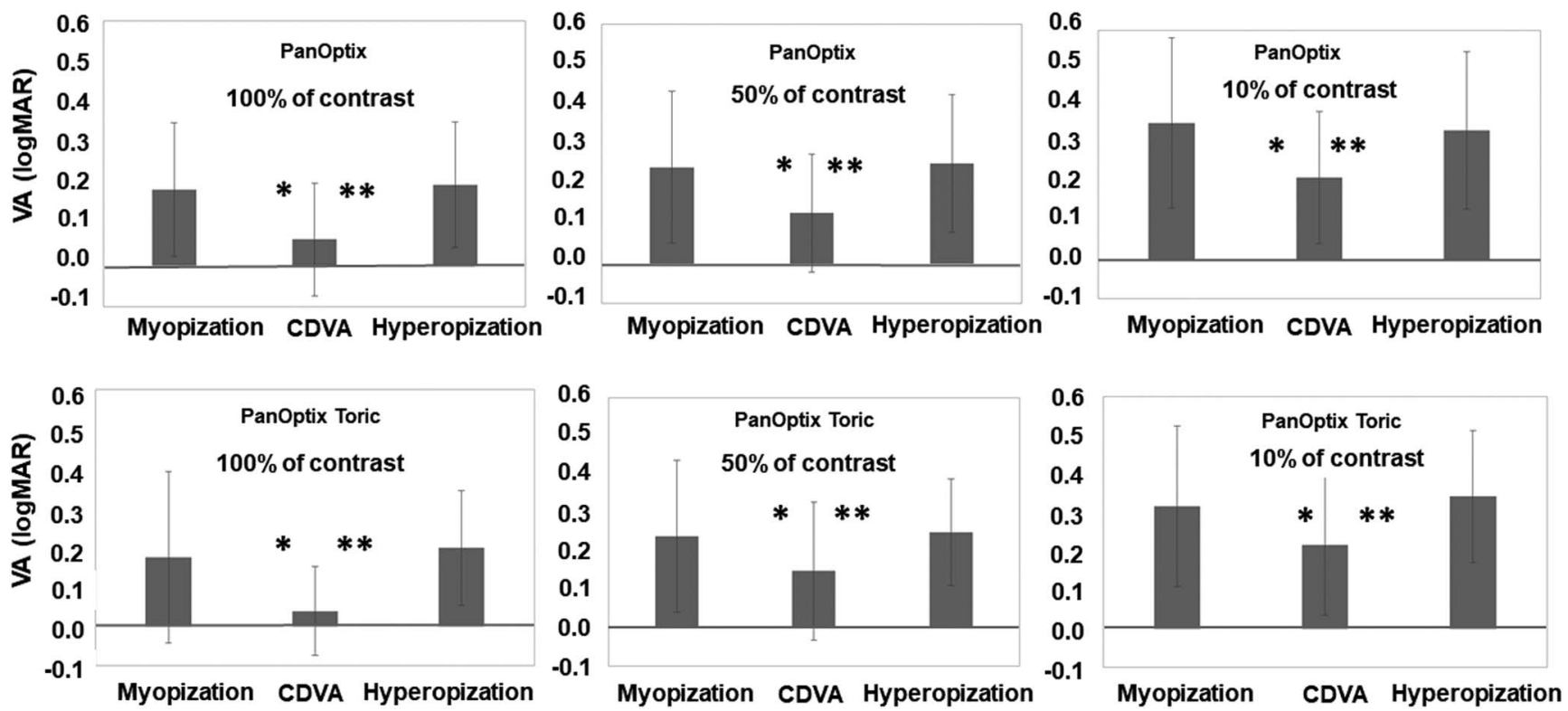

FIG. 1. Visual acuity at $100 \%, 50 \%$, and $10 \%$ of contrast with best-corrected distance visual acuity (CDVA) and with +0.50 and -0.50 diopters (D) defocus for the PanOptix (Top) and PanOptix Toric groups (Bottom). *Statistically significant differences between the best distance correction situation and $+0.50 \mathrm{D}$ of defocus (myopization). ${ }^{*}$ Statistically significant differences between the best distance correction situation and $-0.50 \mathrm{D}$ of defocus (hyperopization). 

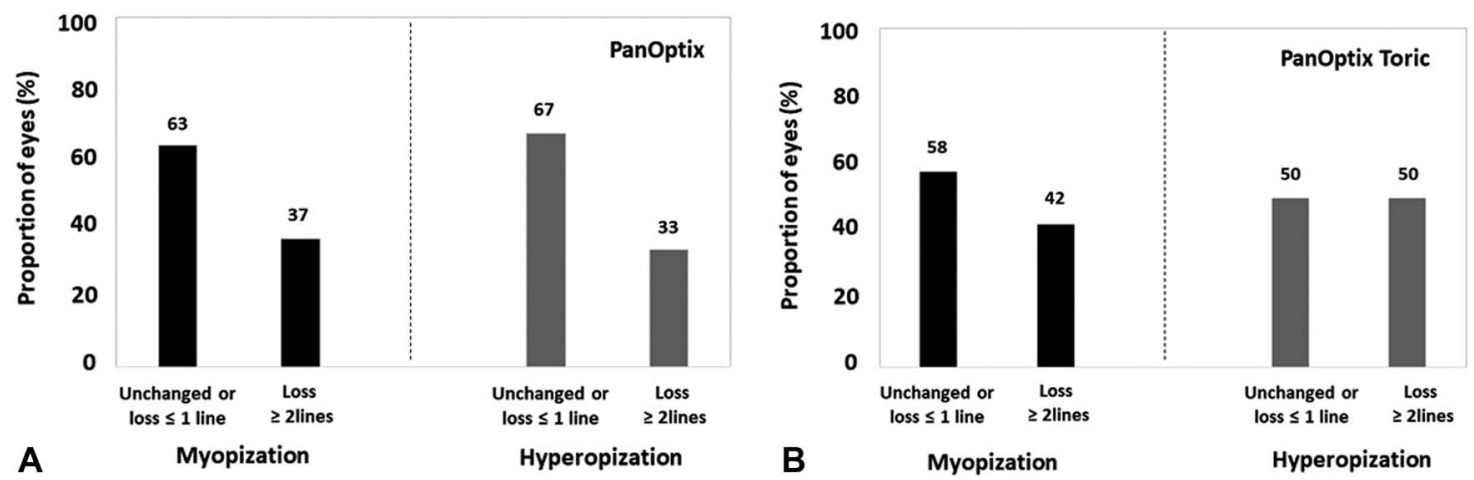

FIG. 2. Proportion of eyes in which the visual acuity (VA) at distance and at $100 \%$ of contrast remained unchanged or dropped only one line and those eyes in which VA decreased $\geq 2$ lines of vision for the best distance correction situation, with $+0.50 \mathrm{D}$ and $-0.50 \mathrm{D}$ of defocus. (A) PanOptix group. (B) PanOptix Toric group.

function correctly or the presence of halos and glare. ${ }^{18}$ These complaints might be related to the presence of small refractive errors. Therefore, the current study assessed the impact of both positive and negative residual refractive errors on visual quality and halo perception in patients implanted with trifocal and trifocal toric IOLs.

We found that distance VA dropped as expected with lower contrast settings, with better values with the best distance correction compared to small defocus situations $( \pm 0.50 \mathrm{D})$. It should be noted that VA showed similar drops when a positive or negative residual refractive error of $0.50 \mathrm{D}$ is induced in both groups and for all contrast settings, reflecting the importance of avoiding residual refractive errors when trifocal IOLs are implanted. This agrees with a previous study that also showed a worsening of high contrast VA values when some positive or negative refractive error is induced in patients implanted with the same trifocal IOL. ${ }^{17}$

In addition, to better discern the impact of the type of overrefraction, we analyzed the proportion of patients who undergo a significant VA loss: when a positive defocus of $+0.50 \mathrm{D}$ is induced (myopization), the proportion of eyes that lost $\geq 2$ lines was $37 \%$, with $33 \%$ of eyes losing $\geq 2$ lines when a negative defocus of $-0.50 \mathrm{D}$ (hyperopization) was induced in the PanOptix group. For the PanOptix Toric group, the percentage of eyes that lost $\geq 2$ lines of vision was $42 \%$ and $50 \%$ after myopization and hyperopization, respectively. Therefore, regardless of the type of defocus, a similar and substantial proportion of patients could experience a deterioration in VA with low residual refractive errors.

It must be taken into account that although we have found that residual refractive errors can lead to a deterioration in distance VA with trifocal IOLS, the impact of positive or negative overrefractions should not affect VA at intermediate and/or near vision but only induce a displacement (forward or backward) of all focal points of the defocus curve. ${ }^{1-7}$ As a consequence, residual refractive errors with trifocal IOLs would modify the preferred distances for intermediate or near activities but not the vision at these distances.

To evaluate more comprehensively visual quality, CSF was also measured in the three situations for both groups. It should be noted that for low frequencies (three cycles per degrees), that is, low detail-demanding visual situations, no differences were found
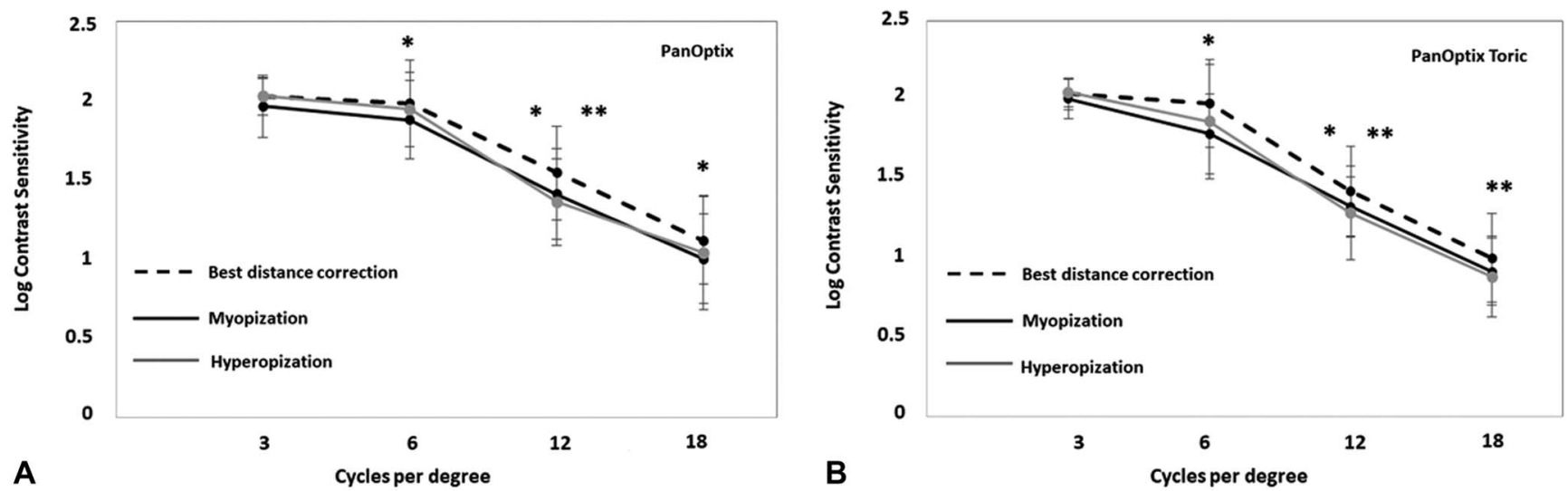

FIG. 3. Contrast sensitivity function (CSF) under photopic conditions $\left(85 \mathrm{~cd} / \mathrm{m}^{2}\right) 3$ months after the cataract surgery for the three refractive situations (best distance correction, $+0.50 \mathrm{D}$ and $-0.50 \mathrm{D}$ defocus). (A) PanOptix group. (B) PanOptix Toric group. *Statistically significant differences between the best distance correction situation and $+0.50 \mathrm{D}$ defocus (myopization). ${ }^{* *}$ Statistically significant differences between the best distance correction situation and $-0.50 \mathrm{D}$ defocus (hyperopization). 


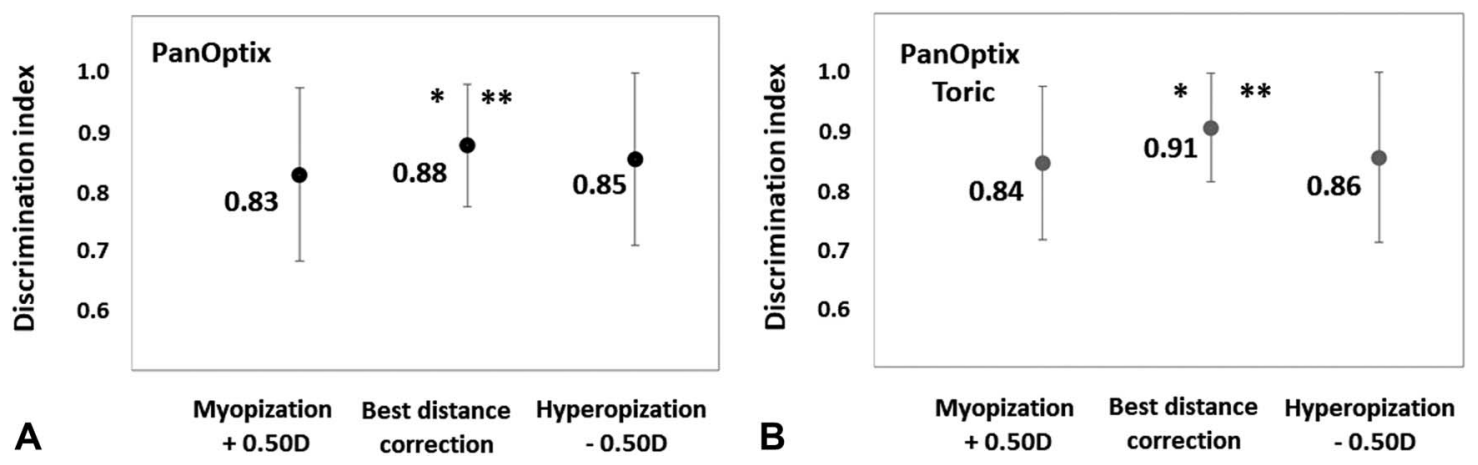

FIG. 4. Discrimination index (halo effect) for the three refractive situations (best distance correction, $+0.50 \mathrm{D}$ and $-0.50 \mathrm{D}$ defocus). A higher discrimination index reflects a lower halo perception. (A) PanOptix group. (B) PanOptix Toric group. *Statistically significant differences between the best distance correction situation and $+0.50 \mathrm{D}$ defocus (myopization). ${ }^{* *}$ Statistically significant differences between the best distance correction situation and $-0.50 \mathrm{D}$ defocus (hyperopization).

among the three situations and for both IOLs groups. As the frequencies become higher and the resolution of fine details is more demanding, the results showed more variability. The overall trend at higher frequencies shows that CSF is better when no residual refractive error is induced. Thus, for gross visual details, some degree of residual refractive error seems to be negligible, whereas fine detail resolution could be affected even with low residual refractive errors, whether they are positive or negative.

Another issue in patients who have been implanted with multifocal IOLs is the impact of photic phenomena. We found that the mean halo effect was lower (higher discrimination index) when no residual refractive error is induced, with a higher halo perception with defocus, which again is similar both for negative and positive defocus. A previous study performed with an optical bench suggested that in trifocal IOLs, the halo in distance vision is produced by the joint contribution of the out-of-focus images corresponding to the intermediate and near add powers. ${ }^{15}$ Therefore, the displacement (backward or forward) of the three foci induced by residual refraction could modify their contribution to halo perception at distance vision. However, the averaged results of the discrimination index showed that although low overrefractions increase halo perception, there are no significant differences between positive or negative defocus.
Alba Bueno et al. ${ }^{15}$ also analyzed psychophysical assessment of halo perception and clinical subjective complaints of patients due to photic phenomena. The authors reported that a $10 \%$ decrease in the discrimination index was correlated with significant subjective patient complaints. To further measure the impact of the type of defocus on halo perception, the proportion of patients who lost $\geq 10 \%$ of the discrimination index and also the proportion of eyes that lost $\geq 10 \%$ of the discrimination index without a significant VA were analyzed. With this analysis, the proportion of patients who might complain only due to photic phenomena would be determined independently from those whose visual complaints might be related to a significant VA loss. Our results found that a low defocus of $\pm 0.50 \mathrm{D}$ could have a significant impact on halo perception in between $11 \%$ and $17 \%$ of the patients implanted with trifocal IOLs with no significant changes in distance VA. Therefore in a clinical setting, we must bear in mind that complaints about photic phenomena might be related to the presence of low residual refractive errors that seem to have no influence on VA.

In the current study, no postoperative misalignments of the IOLs were found and patients presented regular corneal profiles. Intraocular lens decentration, tilt, or rotation might further compromise the optical performance of the IOLs and patient visual quality. ${ }^{19-22}$ Hence, it would be interesting to analyze the effect of

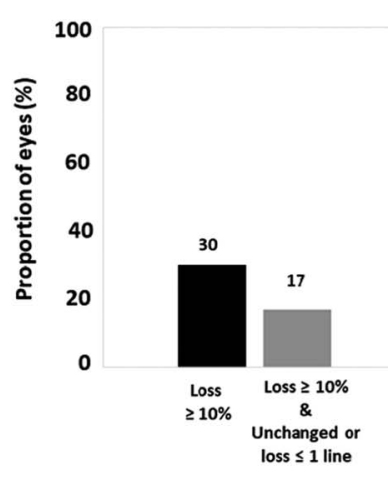

A

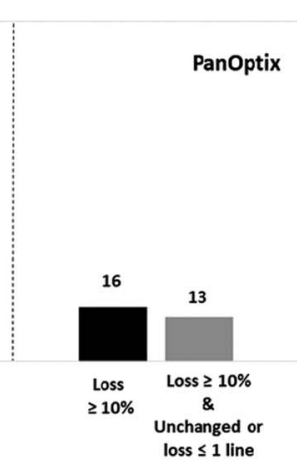

Hyperopization

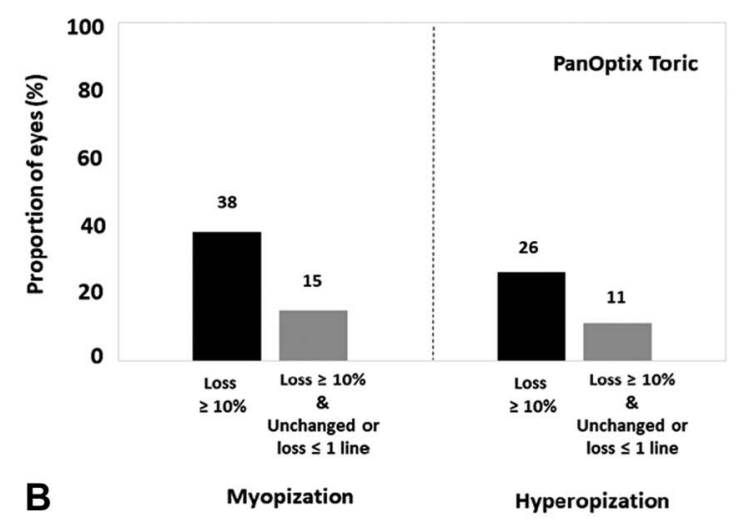

FIG. 5. Proportion of patients who showed a loss $\geq 10 \%$ of discrimination index and those in whom the index loss $\geq 10 \%$ while their visual acuity remained unchanged or loss $\leq 1$ lines with $+0.50 \mathrm{D}$ or $-0.50 \mathrm{D}$ of defocus. (A) PanOptix group. (B) PanOptix Toric group. 
the combination of misalignments and residual refractive errors in patients with trifocal IOLs. At the same time, dramatic changes on corneal profiles, such as those present in some patients with previous corneal refractive surgery, could also deteriorate their visual quality and/or halo perception. ${ }^{23,24}$ In upcoming years, a large number of patients who have undergone corneal refractive surgery will develop cataracts and will probably seek spectacle independence; therefore, studies to assess the potential effects of residual refractive errors in these patients after trifocal or trifocal toric IOL implantation will be of great interest.

In conclusion, the results of this study suggest that, regardless of their nature, low residual refractive errors might be tolerated by most patients implanted with trifocal or trifocal toric IOLs. However, a significant number of patients will probably complain due to decreased visual quality, increased photic phenomena, or a combination of both situations. Therefore, residual refractive errors within $\pm 0.50 \mathrm{D}$ after surgery should not always be considered a refractive success after premium IOL implantation.

\section{REFERENCES}

1. Rementería-Capelo LA, Contreras I, García-Pérez JL, et al. Visual quality and patient satisfaction with a trifocal intraocular lens and its new toric version. J Cataract Refract Surg 2019;45:1584-1590.

2. Poyales F, Garzon N. Comparison of 3-month visual outcomes of a spherical and a toric trifocal intraocular lens. J Cataract Refract Surg 2019;45: 135-145.

3. Piovella M, Colonval S, Kapp A, et al. Patient outcomes following implantation with a trifocal toric IOL: Twelve-month prospective multicentre study. Eye (Lond) 2019; 33:144-153.

4. Böhm M, Hemkeppler E, Herzog M, et al. Comparison of a panfocal and trifocal diffractive intraocular lens after femtosecond laser-assisted lens surgery. J Cataract Refract Surg 2018; 44:1454-1462.

5. Alfonso JF, Fernández-Vega-Cueto L, Fernández-Vega L, et al. Visual function after implantation of a presbyopia-correcting trifocal intraocular lens Ophthalmic Res 2020;63:152-164.

6. Kohnen T, Herzog M, Hemkeppler E, et al. Visual performance of a quadrifocal (trifocal) intraocular lens following removal of the crystalline lens. Am J Ophthalmol 2017;184:52-62.

7. Cochener B, Boutillier G, Lamard M, et al. A comparative evaluation of a new generation of diffractive trifocal and extended depth of focus intraocular lenses. J Refract Surg 2018;34:507-514.

8. Alio JL, Abdelghany AA, Fernández-Buenaga R. Enhancements after cataract surgery. Curr Opin Ophthalmol 2015;26:50-55.
9. Alfonso JF, Fernández-Vega L, Montés-Micó R, et al. Femtosecond laser for residual refractive error correction after refractive lens exchange with multifocal intraocular lens implantation. Am J Ophthalmol 2008;146: 244-250.

10. Bach M. The Freiburg Visual Acuity Test-automatic measurement of visual acuity. Optom Vis Sci 1996;73:49-53.

11. Bach M. The Freiburg visual acuity test-variability unchanged by post-hoc re-analysis. Graefes Arch Clin Exp Ophthalmol 2007;245: 965-971.

12. Castro JJ, Ortiz C, Pozo AM, et al. A visual test based on a freeware software for quantifying and displaying nightvision disturbances: Study in subjects after alcohol consumption. Theor Biol Med Model 2014;11: S1.

13. Castro JJ, Jimenez JR, Ortiz C, et al. New testing software for quantifying discrimination capacity in subjects with ocular pathologies. $J$ Biomed Opt 2011;16:015001.

14. Anera RG, Castro JJ, Jimenez JR, et al. Optical quality and visual discrimination capacity after myopic LASIK with a standard and aspheric ablation profile. J Refract Surg 2011;27:597-601.

15. Alba-Bueno F, Garzón N, Vega F, et al. Patient-perceived and laboratorymeasured halos associated with diffractive bifocal and trifocal intraocular lenses. Curr Eye Res 2018;43:35-42.

16. Carballo-Alvarez J, Vazquez-Molini JM, Sanz-Fernandez JC, et al. Visual outcomes after bilateral trifocal diffractive intraocular lens implantation. BMC Ophthalmol 2015;15:26.

17. Hayashi K, Sato T, Igarashi C, et al. Effect of spherical equivalent error on visual acuity at various distances in eyes with a trifocal intraocular lens. $J$ Refract Surg 2019;35:274-279.

18. Cao K, Friedman DS, Jin S, et al. Multifocal versus monofocal intraocular lenses for age-related cataract patients: A system review and meta-analysis based on randomized controlled trials. Surv Ophthalmol 2019;64:647-658

19. Baumeister M, Buhren J, Kohnen T. Tilt and decentration of spherical and aspheric intraocular lenses: Effect on higher-order aberrations. J Cataract Refract Surg 2009;35:1006-1012.

20. Fujikado T, Saika M. Evaluation of actual retinal images produced by misaligned aspheric intraocular lenses in a model eye. Clin Ophthalmol 2014;8:2415-2423.

21. Tognetto D, Perrotta AA, Bauci F, et al. Quality of images with toric intraocular lenses. J Cataract Refract Surg 2018;44:376-381.

22. Garzón N, Poyales F, de Zárate BO, et al. Evaluation of rotation and visual outcomes after implantation of monofocal and multifocal toric intraocular lenses. J Refract Surg 2015;31:90-97.

23. Lee YC, Hu FR, Wang IJ. Quality of vision after laser in situ keratomileusis: Influence of dioptric correction and pupil size on visual function. J Cataract Refract Surg 2003;29:769-777.

24. Villa C, Gutiérrez R, Jiménez JR, et al. Night vision disturbances after successful LASIK surgery. Br J Ophthalmol 2007;91:1031-1037. 